\title{
Similaridade florística entre estratos da vegetação em quatro Florestas Estacionais Deciduais na bacia do Rio São Francisco
}

Floristic similarity between strata of vegetation in four Deciduous Seasonal Forest in São Francisco River

\author{
Anne Priscila Dias Gonzaga ${ }^{1,4}$, José Roberto Rodrigues Pinto ${ }^{2}$, Evandro Luiz Mendonça Machado ${ }^{3}$ \\ \& Jeanine Maria Felfili ${ }^{2, \dagger}$
}

\begin{abstract}
Resumo
Este estudo descreveu e comparou a similaridade florística de três estratos de quatro fragmentos de Floresta Estacional Decidual. Os fragmentos estudados estão distribuídos na Bacia do São Francisco, três em Minas Gerais e um na Bahia. A amostragem da vegetação seguiu o protocolo da Rede de Parcelas Permanentes nos biomas Cerrado e Pantanal para os estratos arbóreo, de arvoretas e juvenil. Foram contabilizadas a riqueza de espécies e famílias em cada área e estrato. A similaridade entre os estratos de cada área foi obtida por diagramas de Venn e índices de Jaccard e Czekanowski, já para a análise da similaridade entre os fragmentos, em cada estrato, foram calculados apenas os dois índices. Os quatro fragmentos e os três estratos apresentaram baixa relação florística, ou seja, baixo compartilhamento de espécies. As diferenças entre os estratos podem ser reflexo de variações ambientais ao longo do tempo, assim como da capacidade de reprodução das espécies dos estratos superiores. Já as diferenças entre as áreas devem-se à distribuição geográfica, que gera variações ambientais, as quais podem estar favorecendo a ocorrência de espécies adaptadas às condições de cada fragmento, contribuindo para uma composição florística diferenciada. Além disso, os contatos vegetacionais (ecótonos) provavelmente também contribuíram para as diferenças florísticas observadas.

Palavras-chave: padrões florísticos, heterogeneidade ambiental, variação entre estratos.
\end{abstract}

\begin{abstract}
This study described and compared the floristic similarity in four Deciduous Seasonal Forest fragments, and assessed the floristic relations between the tree (adult), the treelet and the sapling stratum. The fragments studied are distributed in Minas Gerais State and in Bahia State, Brazil. The vegetation sampling method followed the protocol of the "Rede de Parcelas Permanentes nos biomas Cerrado e Pantanal" for the tree, treelet and sapling strata. The floristic similarity between the fragments was obtained based on Jaccard and Czekanowski indices. Besides the similarity indices, Venn diagrams were drawn for the strata within each area. The four fragments and the three strata of each fragment presented low degrees of species sharing. The floristic differences among the strata may be due to environmental variations throughout time as well as the reproductive capacity of the species differentiated among the years. Besides the geographic distance (neutral theory), the environmental variations between the areas and the vegetation contacts (ecotones) with other biomes may have favored the occurrence of species adapted to the conditions of each fragment, which contributed to the distinct floristic composition. Key words: floristic patterns, environmental heterogeneity, variation between strata.
\end{abstract}

\section{Introdução}

No Brasil, as Florestas Estacionais Deciduais ou matas secas ocorrem naturalmente na forma de machas, distribuídas ao longo do Brasil Central (Mato Grosso do Sul, Mato Grosso, Goiás e Tocantins), norte de Minas Gerais e Bahia (Rizzini 1997). Tais florestas também podem ser encontradas na região sul do Brasil (IBGE 2004), porém, estas apresentam outra condição ambiental (baixas temperaturas) ocasionando a deciduidade foliar (Oliveira-Filho 2009). De acordo com Prado \& Gibbs (1993), a distribuição em manchas desta fisionomia florestal,

\footnotetext{
Este artigo possui material adicional em sua versão eletrônica.

${ }^{1}$ Universidade Federal dos Vales do Jequitinhonha e Mucuri, Curso de Geografia, 39100-000, Diamantina, MG, Brasil

${ }^{2}$ Universidade de Brasília, Depto. Engenharia Florestal, 70919-970 Brasília, DF, Brasil.

${ }^{3}$ Universidade Federal dos Vales do Jequitinhonha e Mucuri, Depto. Engenharia Florestal, 39100-000, Diamantina, MG, Brasil.

${ }^{4}$ Autor para correspondência: diaspri@gmail.com

${ }^{\dagger}$ In memoriam
} 
provavelmente, indica que seriam remanescentes de uma antiga floresta contínua, que conectava a Caatinga aos Chacos argentinos durante o Pleistoceno, a cerca de 10.000 anos antes do presente.

Entre as principais características físicas e ambientais que distinguem a mata seca das demais formações florestais tropicais, destaca-se a sua frequente ocorrência sob afloramentos calcários e, em virtude desta associação, a existência de solos rasos, porém com elevada disponibilidade de nutrientes (Murphy \& Lugo 1986; Felfili et al. 1998; Werneck et al. 2000; Silva \& Scariot 2004a, b). É importante ressaltar que, apesar de rara, as Florestas Estacionais Deciduais também podem ocorrer sobre relevo plano, onde seus solos são mais profundos (Sampaio 2006). Outros aspectos importantes são a elevada caducifolia apresentada por seus indivíduos, o que teria originado o seu nome popular "mata seca", a grande amplitude climática ao longo da sua distribuição (Santos et al. 2007a), a qual está relacionada ao fato deste tipo fisionômico se distribuir ao longo dos biomas da Mata Atlântica, do Cerrado e da Caatinga (IBGE 2004), e a sua dissociação de cursos d'água (Ribeiro \& Walter 2008).

De acordo com Espirito-Santo et al. (2008), as Florestais Estacionais Deciduais ocupam 27.367,815 ha, o que representa pouco mais de 3\% do território nacional. Apesar da reduzida área de ocorrência, esta fisionomia é considerada portadora de significativa diversidade biológica (Gentry 1995; Silva \& Scariot 2004a, b; Felfili et al. 2007) e elevada diversidade de formas de vida (Medina 1995; Melo 2008). Porém, apesar da sua considerável importância ecológica e diversidade biológica, tanto florística como faunística, este tipo de vegetação se encontra entre as fisionomias brasileiras menos conhecidas (Gonzaga et al. 2007; Santos et al. 2007a).

Segundo Espirito-Santo et al. (2008), devido à ausência de estudos detalhados, as taxas de desmatamento e o real estado de degradação dos fragmentos de Floresta Estacional Decidual, até o momento, não foram precisamente quantificados. Por esta razão, semelhante ao ocorrido na maior parte da vegetação brasileira, grandes porções do patrimônio biológico das Florestas Estacionais Deciduais estão sendo perdidas por meio da ação humana, sem se obter, ao menos, o conhecimento ecológico básico destes ambientes.

Diante deste cenário, boa parte do conhecimento acerca das Florestas Estacionais Deciduais realizados no Brasil advém dos levantamentos florísticos e fitossociológicos, como os de Felfili et al. (1998), Silva \& Scariot (2003, 2004a,b), Nascimento et al. (2004), Salis et al. (2004), Santos et al. (2007b), ou de similaridades florísticas entre diferentes áreas (Santos \& Vieira 2006; Santos et al. 2007a). Por outro lado, poucos estudos, a exemplo do presente, compararam a florística de fragmentos de Floresta Estacional Decidual numa escala geográfica, ou fizeram comparações entre os estratos da vegetação, por exemplo, entre regenerantes e a comunidade adulta (Medeiros et al. 2007).

Desta forma, o presente estudo objetivou descrever as características florísticas em três estratos da vegetação (arbóreo, arvoretas e juvenis), avaliados em quatro fragmentos de Floresta Estacional Decidual, amostrados na bacia do Rio São Francisco. Para tanto, buscou-se responder a seguinte pergunta: existe semelhança florística entre os estratos adulto e regenerante (arvoretas e juvenis) nas comunidades de Floresta Estacional Decidual?

\section{Material e Métodos}

Foram amostrados quatro fragmentos de Floresta Estacional Decidual (sensu Veloso et al. 1991) distribuídos ao longo da bacia hidrográfica do Rio São Francisco, sendo três em Minas Gerais (municípios de Arcos, Paracatu e Peruaçu) e outro na Bahia (município de Coribe). A extensão deste trabalho é compreendida pelas coordenadas geográficas $13^{\circ} 29^{\prime}-20^{\circ} 17^{\prime} \mathrm{S}$ e $44^{\circ} 14^{\prime}-46^{\circ} 49^{\prime} \mathrm{W}$ (Fig. 1, Tab. 1).

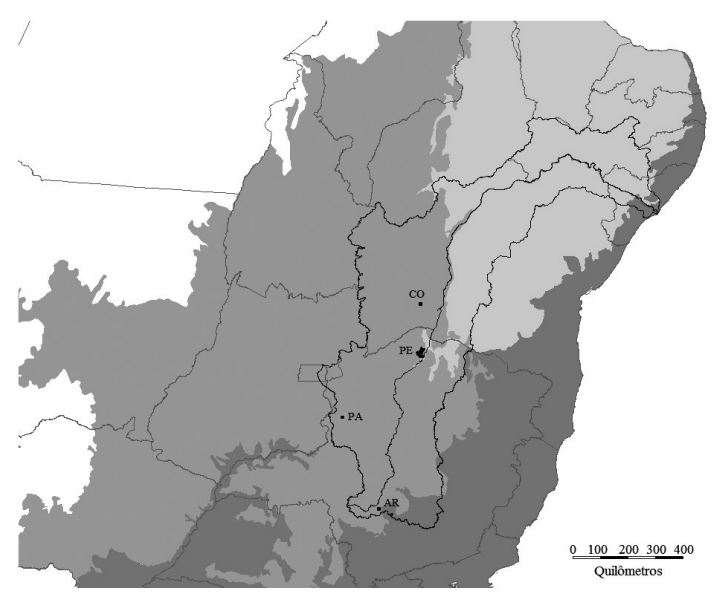

Figura 1 - Localização dos quatro fragmentos de Floresta Estacional Decidual amostrados, com detalhe para os limites da bacia hidrográfica do Rio São Francisco e dos biomas (sensu MMA 2009) Caatinga; Cerrado; Mata Atlântica. Onde: $\mathrm{AR}=\operatorname{Arcos}(\mathrm{MG}) ; \mathrm{PA}=$ Paracatu $(\mathrm{MG}) ; \mathrm{PE}=$ Peruaçu (MG) e $\mathrm{CO}=$ Coribe $(\mathrm{BA})$.

Figure 1 - Location of the four Deciduous Seasonal Forest fragments sampled, pointing out the limits of São Francisco River watershed and the Caatinga, Cerrado and Atlantic Forest biomes (sensu MMA 2009). Where: $A R=\operatorname{Arcos}(M G)$; $\mathrm{PA}=$ Paracatu $(\mathrm{MG}) ; \mathrm{PE}=$ Peruaçu $(\mathrm{MG})$ e $\mathrm{CO}=$ Coribe $(\mathrm{BA})$. 
Tabela 1 - Informações geográficas e ambientais dos quatro fragmentos de Floresta Estacional Decidual utilizados nas análises comparativas deste estudo.

Table 1 - Geographical and environmental information of the four Deciduous Seasonal Forest fragments used in the comparative analyses.

\begin{tabular}{lcccc}
\hline & Arcos (MG) & Paracatu (MG) & Peruaçu (MG) & Coribe (BA) \\
\hline Município (estado) & Arcos (Minas Gerais) & Paracatu (Minas Gerais) & $\begin{array}{l}\text { Januária / Itacarambi / São João } \\
\text { das Missões (Minas Gerais) }\end{array}$ & $\begin{array}{l}\text { São Félix do } \\
\text { Coribe (Bahia) }\end{array}$ \\
Propriedade & $\begin{array}{l}\text { Estação Ecológica de } \\
\text { Corumbá (IEF) }\end{array}$ & $\begin{array}{l}\text { Propriedade privada } \\
\text { Parque Nacional Carvernas do }\end{array}$ & $\begin{array}{l}\text { Propriedade } \\
\text { privada }\end{array}$ \\
Latitude (S) & $20^{\circ} 17^{\prime}$ & $17^{\circ} 13^{\prime}$ & $14^{\circ} 54^{\prime}$ & $1^{\circ} 29^{\prime}$ \\
Longitude (W) & $45^{\circ} 37^{\prime}$ & $46^{\circ} 49^{\prime}$ & $44^{\circ} 22^{\prime}$ & $44^{\circ} 14^{\prime}$ \\
Altitude & 819 & 626 & 696 & 533 \\
Área (ha) & 100 & 100 & 200 & 50 \\
Clima* & $\mathrm{Cwa}$ & Aw & Aw & Aw \\
Temperatura Média Anual $\left({ }^{\circ} \mathrm{C}\right)^{\#}$ & 20,7 & 22,0 & 26,3 & 24,4 \\
Precipitação Média Anual $(\mathrm{mm})^{\#}$ & 1.600 & 1.350 & 947 & 900 \\
\hline
\end{tabular}

"IBGE (2002); " INMET (2010)

Segundo IBGE (2002), o clima da região é classificado como do tipo Aw (clima tropical) e Cwa (clima subtropical úmido). O primeiro apresenta estação seca bem definida, onde pelo menos um dos meses do ano tem precipitação média total inferior a $60 \mathrm{~mm}$ e temperatura média mensal nunca inferior a $18^{\circ} \mathrm{C}$. Já Para o clima tipo Cwa a temperatura varia regularmente ao longo do ano, apresentando verões brandos e suaves, com estiagens de inverno (Tab. 1).

De acordo com a classificação da vegetação brasileira (IBGE 2004), as quatro localidades estudadas possuem como vegetação dominante a Floresta Estacional Decidual. Porém, as mesmas estão inseridas em distintas regiões fitogeográficas, como áreas core do Cerrado (Paracatu, MG), de ecótono Cerrado/Caatinga (município de São Félix do Coribe, BA e Parque Nacional Cavernas do Peruaçu, MG), além de áreas com forte influência da Mata Atlântica (Estação Ecológica de Corumbá, MG) (Fig. 1).

Para amostrar a vegetação foi instalada uma rede de parcelas, seguindo o protocolo da Rede de Parcelas Permanentes nos biomas Cerrado e Pantanal (Felfili et al. 2005). Para a amostragem do estrato arbóreo foram alocadas aleatoriamente 25 parcelas de $20 \times 20 \mathrm{~m}$ (Fig. 2), em cada uma das quatro áreas. Foram amostrados todos os indivíduos arbóreos contidos no interior das unidades amostrais, com diâmetro à altura do peito $(\mathrm{DAP}) \geq 5 \mathrm{~cm}$, exceto indivíduos mortos, lianas e trepadeiras.

Para amostragem das arvoretas foi alocada no canto inferior esquerdo de cada parcela de 20 $\times 20 \mathrm{~m}$ uma parcela de $5 \times 5 \mathrm{~m}$ (Fig. 2), conforme método sugerido por Felfili et al. (2005). Nestas parcelas, foram amostrados todos os indivíduos arbustivo-arbóreos com altura $>1,0 \mathrm{~m}$ e DAP $<5,0$ $\mathrm{cm}$, conforme adotado por Pinto \& Hay (2005) e Felfili et al. (2005). Para o estrato das juvenis foram alocadas, no canto inferior esquerdo de cada parcela de $5 \times 5 \mathrm{~m}$, uma sub-parcela $2 \times 2 \mathrm{~m}$ (Fig. 2). Nestas parcelas foram amostrados todos os indivíduos com altura < 1,0 m (Pinto \& Hay 2005; Felfili et al. 2005).

O material botânico coletado foi depositado no Herbário Dendrológico Jeanine Felfili (HDJF), da Universidade Federal dos Vales do Jequitinhonha e Mucuri (UFVJM). As espécies foram classificadas em famílias de acordo com o Grupo Filogenético das Angiospermas (APG III 2009). As sinonímias e grafia dos nomes científicos foram consultadas na lista de espécies da flora do Brasil (Forzza et al. 2010).

Para avaliar a composição florística dos fragmentos amostrados, foram contabilizadas a riqueza de espécies e famílias por área e por estrato. Além disso, foi analisada a similaridade de espécies entre os estratos e foram confeccionados, para cada área, diagramas de Venn, com base na presença e ausência das espécies nos estratos avaliados. Foram calculados os índices de similaridade qualitativos (Jaccard), entre os estratos dentro da mesma área e entre as diferentes áreas. Índices de similaridade quantitativos (Czekanowski) foram calculados apenas entre os estratos de cada área (Kent \& Coker 1992; Mueller-Dombois \& Ellenberg 2002). 


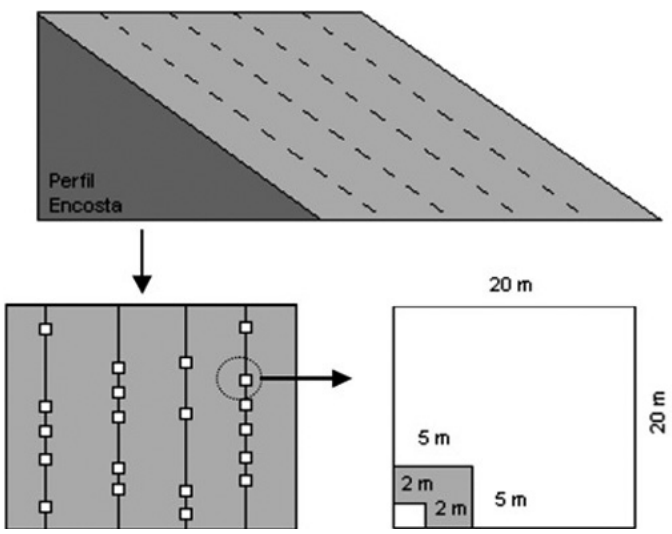

Figura 2-Esquema da amostragem dos estratos davegetação em Florestas Estacionais Deciduais(Fonte: Felfili et al. 2005). Figure 2 - Sampling scheme of the three vegetation strata used in Deciduous Seasonal Forest fragments (Source: Felfili et al. 2005).

\section{Resultados}

Quando analisados em conjunto os três estratos dos quatro fragmentos de Floresta Estacional Decidual amostrados, a riqueza total encontrada foi de 204 espécies arbustivo-arbóreas, pertencentes a 45 famílias (Apêndice). Neste conjunto se somariam mais 19 morfoespécies as quais, por não se encontrarem identificadas ao nível de espécie até o momento, foram excluídas das análises.

A análise conjunta da composição florística em nível de família, para todas as áreas e estratos estudados, mostrou que Fabaceae foi a que apresentou a maior riqueza (51 espécies), seguida por Myrtaceae (15), Malvaceae (12) e Rubiaceae (10), as quais juntas representam $43,13 \%$ da riqueza em espécies registradas nos quatro fragmentos. Neste conjunto de dados, foram observadas 18 famílias (40\% da amostragem total) com ocorrência limitada a uma área. Exemplos dessa baixa representatividade são Malpighiaceae, Rhamnaceae, Nyctaginaceae e Lauraceae, dentre outras.

Ao avaliar as áreas separadamente foi possível verificar número similar de famílias entre as mesmas, os quais variam entre 25 e 29 famílias. Porém, quando se analisa a riqueza em espécies verifica-se certa discrepância entre as áreas, onde o fragmento de Paracatu foi o mais rico (90 espécies), seguido por Peruaçu (79), Arcos (73) e Coribe (54).

As diferenças entre as áreas ficaram ainda mais evidentes quando foram considerados os compartimentos da vegetação separadamente. Os fragmentos de Peruaçu e Arcos foram os que apresentaram maior semelhança no número de espécie entre os estratos arbóreo e arvoretas (Arcos: Arbóreo $=46$ espécies; Arvoretas $=48$ e Juvenis = 28; Peruaçu: Arbóreo $=64$ espécies; Arvoretas $=56$ e Juvenis $=34$ ). Já em Coribe houve redução de $50 \%$ no número de espécies entre os estratos (Arbóreo $=43$ espécies; Arvoretas $=20$ e Juvenis $=$ 10). Enquanto que em Paracatu registrou-se maior semelhança no número de espécie entre as arvoretas e as juvenis (Arbóreo $=65$ espécies; Arvoretas $=$ 46 e Juvenis $=40)$.

Em todo o conjunto amostral investigado, apenas cinco espécies $(2,45 \%)$ ocorreram nos quatro fragmentos, as quais foram: Aloysia virgata (Ruiz \& Pav.) A.Juss., Anadenanthera colubrina (Vell.) Brenan, Handroanthus impetiginosus (Mart. ex DC.) Mattos, Luetzelburgia auriculata (Allemão) Ducke e Myracrodruon urundeuva Allemão. Por outro lado, as espécies com ocorrência registrada em apenas uma das áreas amostradas (com um ou mais indivíduos em cada área) representam $67,64 \%$ (138 espécies) de todas as espécies amostradas. Destas, 26 (12,74\%) ocorreram na área de Coribe, $34(16,66 \%)$ no fragmento do Peruaçu, $38(18,62 \%)$ em Arcos e 40 (19,60\%) em Paracatu.

A similaridade florística entre os quatro fragmentos mostrou que os três estratos avaliados apresentam flora muito distinta entre as áreas (Tab. 2). Os valores encontrados para os índices de Jaccard estão abaixo do mínimo utilizado (50\%) para considerar como similares floristicamente (Gauch 1982). Quanto à similaridade florística-estrutural, avaliada pelo índice de Czekanowski, também se verificou baixa semelhança entre as áreas, onde nenhum dos estratos apresentou valor maior que $34 \%$ (Tab. 2). Portanto, assim como a composição florística, o tamanho das populações nas áreas em cada um dos estratos também é bem diferenciada.

A similaridade florística entre os estratos na mesma área também foi baixa. Nas quatro áreas amostradas o número de espécies compartilhadas entre os três estratos avaliados foi inferior a $33 \%$ (Fig. 3). Além disso, quando se analisa o índice de Jaccard entre os estratos fica evidente a existência, em todos os fragmentos, de maior semelhança florística entre os estratos da regeneração (Arvoretas e Juvenis) de uma mesma área, onde a similaridade entre estes variou de 40,74 (Arcos) a 55,17 (Peruaçu) (Fig. 3). De maneira generalizada Peruaçu foi o fragmento que apresentou maior número de espécies compartilhadas entre os estratos, o que resultou nos maiores valores de similaridade (Fig. 3). 
Tabela 2 - Valores de similaridades pelos índices de Jaccard (porção inferior da tabela) e de Czekanowisk (porção superior da tabela) para os três estratos da vegetação avaliados em quatro fragmentos de Floresta Estacional Decidual

Table 2 - Similarity values by Jaccard (lower portion of the table) and Czekanowisk (upper portion of the table) indices for the three vegetation strata assessed in four Deciduous Seasonal Forest fragments

\begin{tabular}{lcccc}
\hline \multirow{2}{*}{ Estratos } & \multicolumn{4}{c}{ Fragmentos } \\
\cline { 2 - 5 } & Arcos & Paracatu & Peruaçu & Coribe \\
\hline $\begin{array}{lcccc}\text { Arbóreo } \\
\text { (DAP } \geq 5,0 \mathrm{~cm})\end{array}$ & & & & \\
Arcos & $*$ & 33,075 & 21,527 & 10,615 \\
Paracatu & 13,265 & $*$ & 21,890 & 14,254 \\
Peruaçu & 12,244 & 19,444 & $*$ & 17,829 \\
Coribe & 8,536 & 8,000 & 17,582 & $*$ \\
$\begin{array}{l}\text { Arvoretas } \\
\text { (altura }>1,0 \mathrm{~m} \text { e }\end{array}$ & & & & \\
DAP $<5,0 \mathrm{~cm})$ & & & & \\
Arcos & $*$ & 16,320 & 10,552 & 1,452 \\
Paracatu & 17,500 & $*$ & 21,718 & 2,637 \\
Peruaçu & 10,638 & 17,241 & $*$ & 6,585 \\
Coribe & 3,030 & 6,451 & 15,151 & $*$ \\
Juvenis \\
(altura $\leq 1,0 \mathrm{~m})$
\end{tabular}

\section{Discussão}

A família Fabaceae obteve maior representatividade, tanto na análise total (quatro áreas e três estratos) quanto com os fragmentos em separado. Esta família também foi considerada abundante na amostragem de vários outros estudos realizados em Florestas Estacionais Deciduais (Ratter et al. 1978; Silva \& Scariot 2004a,b; Nascimento et al. 2004; Salis et al. 2004; Santos \& Vieira 2005; Santos \& Vieira 2006; Fagundes et al. 2007; Santos et al. 2007a; Felfili et al. 2007). Segundo Schrire et al. (2005) e Queiroz (2006), Fabaceae é particularmente rica em espécies típicas de ambientes secos. Gentry (1995) afirma que espécies pertencentes à família Fabaceae, a qual apresenta elevado número de espécies com frutos e sementes anemocóricas, estão bem distribuídas nas Florestas Estacionais Deciduais. Outra possível explicação para a elevada ocorrência de Fabaceae nestas áreas foi a caducifolia desenvolvida para muitas de suas espécies (Queiroz 2009). Esta característica pode ser considerada uma adaptação à sazonalidade hídrica a qual esse tipo de ambiente se encontra submetido, o que parece conferir a estas espécies possibilidades para a ocupação de diferentes hábitats.

As cinco espécies que ocorreram em todos os fragmentos foram consideradas de ampla distribuição geográfica ao longo dos domínios atlântico e savânico (Oliveira-Filho 2006). De fato, Rizzini (1963) e Pedrali (1997) observaram que, em geral, as Florestas Estacionais apresentam flora com grande contribuição de espécies de ampla distribuição. Isto sugere que estas espécies, provavelmente, apresentam grande plasticidade fenotípica, haja vista que as áreas estudadas possuem considerável heterogeneidade ambiental. Resultado semelhante foi observado por Pereira (2008), que estudou quatro fragmentos de Florestas Estacionais Deciduais ao longo do Brasil central e classificou Anadenanthera colubrina e Myracrodruon urundeuva como indiferentes à qualidade do habitat. Além disso, estas espécies são consideradas frequentes nestas formações (Nascimento et al. 2004; Salis et al. 2004; Fagundes et al. 2007) e, por esta razão, algumas delas são consideradas típicas desse tipo de formação (Santos \& Vieira 2006; Fagundes et al. 2007; Santos et al. 2007a).

O baixo número de espécies compartilhadas entre os estratos analisados pode estar relacionado ao fato da atual presença das árvores adultas ser reflexo dos fatores ambientais e bióticos de períodos mais antigos, que atuaram no momento do estabelecimento delas (Oliveira-Filho et al. 1997; Schiavini et al. 2001). Em contrapartida, os eventos ambientais atuais, tais como a disponibilidade hídrica e nutricional do solo, e a chuva de sementes, dentre outros, seriam os responsáveis pela flora e estrutura das populações observadas nos estratos regenerantes (Oliveira \& Felfili 2005). Desta forma, as condições do ambiente atual ou de um passado recente, associadas a fatores bióticos locais, é que definiram as estruturas populacionais e, consequentemente, das comunidades nestes estratos (Oliveira \& Felfili 2005). Além disso, muitas espécies podem ter produzido baixa 

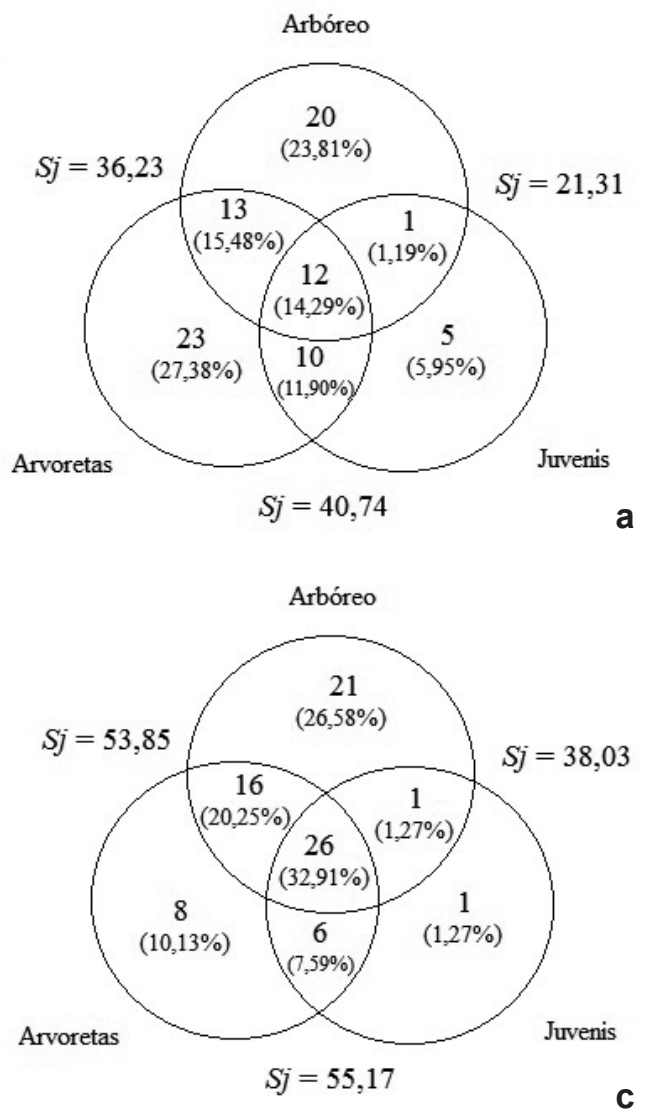
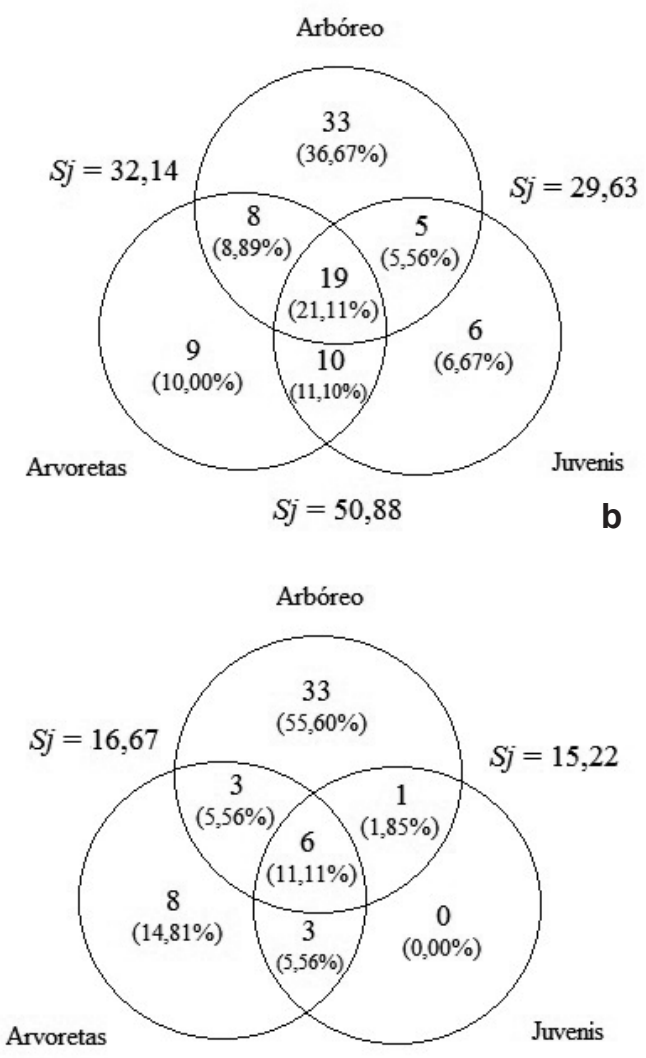

$$
S j=41,86
$$

Figura 3 - Diagramas de Venn produzidos a partir das espécies compartilhadas e exclusivas entre os três estratos da vegetação (Arbóreo - DAP $\geq 5,0 \mathrm{~cm}$; Arvoretas - altura $>1,0 \mathrm{~m}$ e DAP $<5 \mathrm{~cm}$ e Juvenis - altura $\leq 1,0 \mathrm{~m}$ ) amostrados em quatro fragmentos de Floresta Estacional Decidual. $\mathrm{A}=\operatorname{Arcos}(\mathrm{MG}), \mathrm{B}=$ Coribe (BA), $\mathrm{C}=$ Paracatu (MG), $\mathrm{D}=$ Peruaçu $(\mathrm{MG}), \mathrm{Sj}$ = índice de Similaridade de Jaccard entre os pares de estratos indicado.

Figure 3 - Venn Diagrams drawn from exclusive and shared species among the three vegetation strata (tree $-\mathrm{DBH}>5.0 \mathrm{~cm}$; treelet height $>1,0 \mathrm{~m}$ and $\mathrm{DBH}<5.0 \mathrm{~cm}$ and sapling strata - height $<1.0 \mathrm{~m}$ ) sampled in four Deciduous Seasonal Forest fragments. $\mathrm{A}=\mathrm{Arcos}$ (MG), $\mathrm{B}=$ Coribe (BA), $\mathrm{C}=$ Paracatu $(\mathrm{MG})$ and $\mathrm{D}=$ Peruaçu $(\mathrm{MG})$ and $\mathrm{Sj}=$ Jaccard similarity index between pairs of strata indicated.

quantidade de sementes ao longo dos anos, ou mesmo podendo ser descontínuas, o que afetaria a taxa de germinação e o estabelecimento destas espécies na regeneração natural, conforme observado por Schiavini et al. (2001) em outras formações florestais tropicais.

Da mesma forma, os baixos valores dos índices de similaridade de Jaccard e de Czekanowski indicaram elevada dissimilaridade entre os fragmentos quando se analisaram os três estratos avaliados. Tal fato reflete a baixa relação florística entre os estratos adulto e regenerante dentro de cada fragmento, e a existência de variações florísticas dentro de um mesmo estrato entre diferentes áreas amostradas.

Variações geográficas em diferentes escalas podem corresponder a variações ambientais, influenciando nas distribuições diferenciais da abundância das espécies, com consequências para os padrões de diversidade (Stevens \& Carson 2002). Contudo, é importante ressaltar que variações estocásticas podem preponderar sobre as ambientais na distribuição de espécies, porem estas tendem a ocorrer em uma escala local (diversidade alfa) e não em escalas maiores (diversidade beta e, ou gama) (Hubbell 2001).

Outro aspecto importante a ser considerado sobre as diferenças florísticas existentes entre as áreas estudadas é a influência dos contatos vegetacionais (ecótonos) existentes em cada uma delas. As Florestas Estacionais Deciduais brasileiras, por se apresentarem distribuídas descontinuamente em extensa faixa territorial e ocorrerem em grande 
amplitude climática, se encontram sob diferentes fitoregiões (Santos et al. 2007a,b; Pereira 2008). De fato, quando se analisam as espécies de cada fragmento e o local onde as mesmas estão inseridas observou-se que as áreas amostradas apresentaram espécies características dos Biomas nos quais os fragmentos estão inseridos. No caso, por exemplo, do fragmento de Coribe, que se encontra na matriz da Caatinga, é possível notar a presença de espécies típicas desse bioma como Cyrtocarpa caatingae J.D.Mitch. \& Daly e Poincianella pyramidalis (Tul.) L.P.Queiroz (Queiroz 2006; Santos 2009) dentre outras. No remanescente do Peruaçu, que se encontra na transição entre o Cerrado e a Caatinga, verificou-se a presença de Zeyheria tuberculosa que é frequentemente relatada em estudos realizados no bioma Cerrado (Mendonça et al. 2008), além de Amburana cearensis e P. pluviosa que são consideradas endêmicas da Caatinga (Queiroz 2006). De forma semelhante, o fragmento de Paracatu, que está inserido na área core do Cerrado, possui espécies características deste bioma como Dipteryx alata, Curatella americana e J. brasiliana (Marimom \& Lima 2001; Felfili et al. 2002; Mendonça et al. 2008).

No caso do remanescente de Arcos, localizado no contato entre Cerrado e Mata Atlântica, foi observada a presença de espécies destes dois biomas, como Qualea multiflora e Platycyamus regnellii (Mendonça et al. 2008; Machado et al. 2004), sendo a última espécie, considerada peculiar ao ambiente Atlântico. Portanto, a influência da vegetação adjacente na composição florística da Floresta Estacional Decidual parece ter exercido grande influência na composição florística dos fragmentos estudados, fato este também reportado por outros autores que também estudaram Florestas Estacionais Deciduais, como Santos et al. (2007b) e Siqueira et al. (2009).

Os fragmentos de Floresta Estacional Decidual amostrados, assim como os três estratos avaliados, apresentaram fraca relação florística entre si, ou seja, baixo compartilhamento de espécies, tanto quando se comparou o mesmo estrato entre os quatro fragmentos, como os diferentes estratos num mesmo fragmento. Este baixo número de espécies compartilhadas pelos estratos pode ser reflexo tanto da variação nas condições ambientais ao longo do tempo como da própria ecologia de algumas espécies do estrato arbóreo. Além disso, as diferenças florísticas observadas entre os fragmentos podem estar ligadas à distribuição natural das Florestas Estacionais Deciduais, bem como à localização fitogeográfica dos fragmentos, já que a matriz vegetacional destes e os seus vários contatos vegetacionais exerceram considerável influência florística.

\section{Agradecimentos}

Ao Conselho Nacional de Desenvolvimento Científico e Tecnológico (CNPq), a concessão de bolsa de doutorado à primeira autora. Aos que auxiliaram nas identificações das coletas botânicas, em especial a Ary Teixeira de Oliveira Filho, Manoel Cláudio da Silva Júnior, Hisaias de Souza Almeida, Rubens Manoel dos Santos e Newton Rodrigues e aos colaboradores que ajudaram nas coletas de dados.

\section{Referências}

APG III. 2009 An update of the Angiosperm Phylogeny Group classification for the orders and families of flowering plants: APG III. Botanical Journal of the Linnean Society 161: 105-121.

Espírito-Santo, M.M.; Fagundes, M.; Sevilha, A.C.; Scariot, A.O; Sanchez-Azofeifa, G.A.; Noronha, S.E. \& Fernandes, G.W. 2008. Florestas estacionais deciduais brasileiras: distribuição e estado de conservação. MG-Biota 1: 5-13.

Fagundes, L.M.; Carvalho, D.; van den Berg, E.; Melo Marques, J.J.G.S. \& Machado, E.L.M. 2007. Florística e estrutura do estrato arbóreo de dois fragmentos de florestas deciduais às margens do rio Grande, em Alpinópolis e Passos, MG, Brasil. Acta Botanica Brasilica 21: 65-78.

Felfili J.M.; Nogueira, P.E.; Silva-Júnior, M.C.; Marimon, B.S. \& Delitti, W.B.C. 2002. Composição florística e fitossociologia do cerrado sentido restrito no município de Água Boa - MT. Acta Botanica Brasílica 16: 103-112.

Felfili, J.M.; Nascimento, A.R.T.; Meirelles, E.L. \& Fagg, C.W. 2007. Floristic composition and community structure of a seasonally deciduous dry forest on limestone outcrops in Central Brazil. Revista Brasileira de Botânica 30: 375-385.

Felfili, J.M.; Silva Junior, M.C. \& Nogueira, P.E. 1998. Levantamento da vegetação arbórea na região de Nova Xavantina, MT. Boletim do Herbário Ezechias Paulo Heringer 3: 63-81.

Felfili, M.F.; Carvalho, F.A. \& Haidar, R.F. 2005. Manual para monitoramento de parcelas permanentes nos biomas Cerrado e Pantanal. Universidade de Brasília, Departamento de Engenharia Florestal, Brasília. 60p.

Forzza, R.C.; Leitman, P.M.; Costa, A.F.; Carvalho Jr., A.A.; Peixoto, A.L.; Walter, B.M.T.; Bicudo, C.; Zappi, D.; Costa, D.P.; Lleras, E.; Martinelli, G.; Lima, H.C.; Prado, J.; Stehmann, J.R.; Baumgratz, J.F.A.; 
Pirani, J.R.; Sylvestre, L.; Maia, L.C.; Lohmann, L.G.; Queiroz, L.P.; Silveira, M.; Coelho, M.N.; Mamede, M.C.; Bastos, M.N.C.; Morim, M.P.; Barbosa, M.R.; Menezes, M.; Hopkins, M.; Secco, R.; Cavalcanti, T.B. \& Souza, V.C. 2010. Introdução. In: Forzza, R.C. et al. (eds.). Lista de espécies da flora do Brasil. Jardim Botânico do Rio de Janeiro. Disponível em $<$ http://floradobrasil.jbrj.gov.br/2010/>. Acesso em 20 Nov 2011.

Gauch, H.G. 1982. Multivariate analysis in community ecology. Cambridge University Press, Cambridge. $298 \mathrm{p}$.

Gentry, A.H. 1995. Diversity and floristic composition of neotropical dry forests. In: Bullock, S.H.; Mooney, H.A. \& Medina, E. (eds.). Seasonally dry tropical forests. Cambridge University Press, Cambridge. Pp. 146-194.

Gonzaga, A.P.D.; Almeida, H.S.; Nunes, Y.R.F.; Machado, E.L.M. \& D'Angelo Neto, S. 2007. Regeneração Natural da Comunidade Arbórea de dois Fragmentos de Floresta Decidual (Mata seca Calcária) no Município de Montes Claros, MG. Revista Brasileira de Biociências 5: 531-533.

Hubbell, S.P. 2001. The unified neutral theory of biodiversity and biogeography. Princeton University Press, Princeton. 375p.

IBGE - Instituto Brasileiro de Geografia e Estatística. 2002. Mapa de climas do Brasil. Escala 1:5.000.000. IBGE, Rio de Janeiro.

IBGE - Instituto Brasileiro de Geografia e Estatística. 2004. Mapa da vegetação do Brasil. Escala 1:5.000.000. IBGE, Rio de Janeiro.

Kent, M. \& Coker, P. 1992. Vegetation description analyses. Behaven Press, London. 363p.

Machado, E.L.M.; Oliveira-Filho, A.T.; Carvalho, W.A.C.; Souza, J.S.; Borém, R.A.T. \& Botezelli, L. 2004. Análise comparativa da estrutura e flora do compartimento arbóreo-arbustivo de um remanescente florestal na Fazenda Beira Lago, Lavras, MG. Revista Árvore 28: 499-516.

Marimon, B.S. \& Lima, E.S. 2001. Caracterização fitofisionômica e levantamento florístico preliminar no Pantanal dos Rios Mortes-Araguaia, Cocalinho, Mato Grosso, Brasil. Acta Botanica Brasilica 15: 213-229.

Medeiros, M.M.; Felfili, J.M. \& Libano, A.M. 2007. Comparação floristico-estrutural dos estratos de regeneração e adulto em Cerrado sensu stricto no Brasil Central. Cerne 13: 291-298.

Medina, E. 1995. Diversity of life forms of higher plants in neotropical dry forests. In: Bullock, S.H., Mooney, H.A. \& Medina, E. (eds.). Seasonally dry tropical forests. Cambridge University Press, Cambridge. Pp. 221-242.

Melo, P.H.A. 2008. Flora vascular relacionada aos afloramentos de rocha carbonática do interior do Brasil. Dissertação de Mestrado. Universidade Federal de Lavras, Lavras. 79p.
Mendonça, R.C.; Felfili, J.M.; Walter, B.M.T.; Silva-Júnior, M.C.; Rezende, A.B.; Filguerias, T.S.; Nogueira, P.E. \& Fagg, C.W. 2008. Flora vascular do bioma Cerrado: checklist com 12.356 espécies. In: Sano, S.M.; Almeida, S.P. \& Ribeiro, J.F. (eds.). Cerrado: ecologia e flora. Vol. 2. Embrapa Cerrados, Brasília. 1279p.

Mueller-Dombois, D. \& Ellenberg, H. 2002. Aims and methods of vegetation ecology. The Blackburn Press, Caldwell. 548p.

Murphy, P.G \& Lugo, E.A. 1986. Ecology of tropical dry forests. Annual Review of Ecology \& Systematics 17: 67-88.

Nascimento, A.R.T.; Felfili, J.M. \& Meirelles, E.M. 2004. Florística e estrutura da comunidade arbórea de um remanescente de Floresta Estacional Decídua de encosta, Monte Alegre, Goiás, Brasil. Acta Botanica Brasilica 18: 659-669.

Oliveira, E.C.L. \& Felfili, J.M. 2005. Estrutura e dinâmica da regeneração natural de uma mata de galeria no Distrito Federal, Brasil. Acta Botanica Brasílica 19: 801-811.

Oliveira-Filho, A.T. 2006. Catálogo das árvores nativas de Minas Gerais: mapeamento e inventário da flora nativa e dos reflorestamentos de Minas Gerais. Editora UFLA, Lavras. 423p.

Oliveira-Filho, A.T. 2009. Classificação das fitofisionomias da América do Sul cisandina tropical e subtropical: proposta de um novo sistema - prático e flexível - ou uma injeção a mais de caos? Rodriguésia 60: 237-258.

Oliveira-Filho, A.T.; Mello, J.M. \& Scolforo, J.R.S. 1997. Effects of past disturbance and edges on tree community structure and dynamics within a fragment of tropical semideciduous forest in southeastern Brazil over a five-year period (1987-1992). Plant Ecology 131: 45-66.

Pedrali, G. 1997. Florestas secas sobre afloramentos de calcário em Minas Gerais: florística e fisionomia. Bios 5: 81-89.

Pereira, B.S. 2008. Relações vegetação-variáveis ambientais em Florestas Estacionais Decíduas em afloramentos calcários no bioma Cerrado e em zonas de transição com a Caatinga e com Amazônia. Tese de Doutorado. Universidade de Brasília, Brasília, 79p.

Pinto, J.R.R. \& Hay, J.D . 2005. Mudanças florísticas e estruturais na comunidade arbórea de uma floresta de vale no Parque Nacional da Chapada dos Guimarães, Mato Grosso, Brasil. Revista Brasileira de Botânica 28: 523-539.

Prado, D.E. \& Gibbs, P.E. 1993. Patterns of species distributions in the dry seasonal Forest South América. Annals Missouri Botany Garden 80: 902-927.

Queiroz, L.P. 2006. The Brazilian caatinga: phytogeographical patterns inferred from distribution data of the Leguminosae. In: Pennington, R.T.; Lewis, G.P. \& Ratter, J.A. (eds.). Neotropical savannas 
and dry forests: plant diversity, biogeography, and conservation. Taylor \& Francis, Boca Raton. Pp. 113-149.

Queiroz, L.P. 2009. Leguminosas da Caatinga. Universidade Estadual de Feira de Santana, Feira de Santana. 443p.

Ratter, J.A.; Askew, G.P.; Montgomery, R.F. \& Gifford, D.R. 1978. Observations on forests of some mesotrophic soils in central Brazil. Revista Brasileira de Botânica 1: 47-58.

Ribeiro, J.F. \& Walter, B.M.T. 2008. As principais fitofisionomias do bioma Cerrado. In: Sano, S.M.; Almeida, S.P. \& Ribeiro, J.F. (eds.). Cerrado: ecologia e flora. EMBRAPA, Brasília. Pp. 151-199.

Rizzini, C.T. 1963. A flora do cerrado. Análise florística das savanas centrais. In: Ferri, M.G. (ed.). Simpósio sobre o Cerrado. Editora da Universidade de São Paulo, São Paulo. Pp. 126-177.

Rizzini, C.T. 1997. Tratado de fitogeografia do Brasil: aspectos ecológicos, sociológicos e florísticos. Vol. 2. Âmbito Cultural, Rio de Janeiro. 747p.

Salis, S.M.; Silva, M.P.; Mattos, P.P.; Silva, J.S.V.; Pott, V.J. \& Pott, A. 2004. Fitossociologia de remanescentes de floresta estacional decidual em Corumbá, estado do Mato Grosso do Sul, Brasil. Revista Brasileira de Botânica 27:671-684.

Sampaio, A.B. 2006. Recuperação das Florestas Estacionais Deciduais de terrenos planos no norte do Vão do Paranã, GO. Tese de Doutorado. Universidade de Brasília, Brasília, 125p.

Santos, R.M. \& Vieira, F.A. 2005. Estrutura e florística de um trecho de mata ciliar do rio Carinhanha no extremo norte de Minas Gerais, Brasil. Revista Científica Eletrônica de Engenharia Florestal 5: 1-13.

Santos, R.M. \& Vieira, F.A. 2006. Similaridade florística entre formações de Mata Seca e mata de galeria no Parque Municipal da Sapucaia, Montes Claros-MG. Revista Científica Eletrônica de Engenharia Florestal 7: 2-10.

Santos, R.M. 2009. Identidade e relações florísticas da Caatinga Arbórea do Norte de Minas Gerais e sudeste da Bahia. Tese de Doutorado. Universidade Federal de Lavras, Lavras. 118p.

Santos, R.M.; Vieira, F.A.; Fagundes, M.; Nunes, Y.R.F. \& Gusmão, E. 2007a. Riqueza e similaridade florística de oito remanescentes florestais no norte de Minas Gerais, Brasil. Revista Árvore 31: 135-144.
Santos, R.M.; Vieira, F.A.; Gusmão, E. \& Nunes, Y.R.F. 2007b. Florística e estrutura de uma Floresta Estacional Decidual, no Parque Municipal do Sapucaia, Montes Claros (MG). Cerne 13: 248256.

Schiavini, I.; Resende, J.C.F. \& Aquino, F.G. 2001. Dinâmica de populações de espécies arbóreas em mata de galeria e mata mesófila na margem do ribeirão Panga, MG. In: Ribeiro, J.F.; Fonseca, C.E.L. \& Sousa-Silva, J.C. (eds.). Cerrado: caracterização e recuperação de matas de galeria. Embrapa Cerrados, Brasília. Pp. 267-299.

Schrire, B.D.; Lavin, M. \& Lewis, G.P. 2005. Global distribution patterns of the Leguminosae: insights from recent phylogenies. Biologiske Skrifter 55: 375-386.

Silva, L.A. \& Scariot, A. 2003. Composição florística e estrutura da comunidade arbórea em uma floresta estacional decidual em afloramento calcário (Fazenda São José, São Domingos-GO, Bacia do Rio Paraná). Acta Botanica Brasilica 17: 307-315.

Silva, L.A. \& Scariot, A. 2004a. Comunidade arbórea de uma floresta estacional decídua sobre afloramentos calcários na bacia do rio Paranã. Revista Árvore 28: 61-67.

Silva, L.A. \& Scariot, A. 2004b. Composição e estrutura da comunidade arbórea de uma Floresta Estacional Decidual sobre afloramento calcário no Brasil Central. Revista Árvore 28: 69-75.

Siqueira, A.S.; Araujo, G.M. \& Schiavini, I. 2009. Estrutura do componente arbóreo e características edáficas de dois fragmentos de floresta estacional decidual no vale do rio Araguari, MG, Brasil. Acta Botanica Brasilica 23: 10-21.

Stevens, M.H.H. \& Carson, W.P. 2002. Resource quantity, not resource heterogeneity, maintains plant diversity. Ecology Letters 5: 420-426.

Veloso, H.P.; Rangel Filho, A.L.R. \& Lima, J.C.A. 1991. Classificação da vegetação brasileira, adaptada a um sistema universal. IBGE, Rio de Janeiro. 124p.

Werneck, M.S.; Franceschinelli, E.V. \& Tameirão-Neto, E. 2000. Mudanças na florística e estrutura de uma floresta decídua durante um período de quatro anos (1994-1998), na região do Triângulo Mineiro, MG. Revista Brasileira de Botânica 23: 399-411. 


\section{Similaridade florística entre estratos da vegetação em quatro Florestas Estacionais Deciduais na bacia do Rio São Francisco}

Floristic similarity between strata of vegetation in four Deciduous Seasonal Forest in São Francisco River

Anne Priscila Dias Gonzaga, José Roberto Rodrigues Pinto, Evandro Luiz Mendonça Machado \& Jeanine Maria Felfili

Apêndice - Lista das espécies registradas nos quatro fragmentos de Floresta Estacional Decidual amostrados com seus respectivos valores de densidade em cada uma das áreas e dos estratos avaliados. Onde: Arb. $=$ arbóreo (DAP $\geq 5,0 \mathrm{~cm}$ ), Arv. $=$ arvoretas (altura $>1,0 \mathrm{~m})$ e Juv. $=$ juvenis (altura $\leq 1,0 \mathrm{~m}$ ).

Appendix - List of the species surveyed in the four Deciduous Seasonal Forest fragments with their respective density values in each area and strata assessed. Where: Arb. $=$ tree $(\mathrm{DBH}>5.0 \mathrm{~cm})$, Arv. $=$ treelet (height $>1.0 \mathrm{~m})$ e Juv. $=$ sapling strata $($ height $<1.0 \mathrm{~m})$.

\begin{tabular}{|c|c|c|c|c|c|c|c|c|c|c|c|c|}
\hline \multirow{2}{*}{$\begin{array}{l}\text { Família } \\
\text { Espécie }\end{array}$} & \multicolumn{3}{|c|}{$\operatorname{Arcos}(\mathrm{MG})$} & \multicolumn{3}{|c|}{ Paracatu (MG) } & \multicolumn{3}{|c|}{ Peruaçu (MG) } & \multicolumn{3}{|c|}{ Coribe (BA) } \\
\hline & Arb. & Arv. & Juv. & Arb. & Arv. & Juv. & Arb. & Arv. & Juv. & Arb. & Arv. & Juv. \\
\hline \multicolumn{13}{|l|}{ ANACARDIACEAE } \\
\hline Astronium fraxinifolium Schott ex Spreng. & & & & 12 & 2 & 22 & & & & 2 & & \\
\hline Astronium graveolens Jacq. & 1 & & & & & & & & & & & \\
\hline Cyrtocarpa caatingae J.D.Mitch. \& Daly & & & & & & & & & & 22 & 3 & 2 \\
\hline Myracrodruon urundeuva Allemão & 95 & 2 & 1 & 116 & 2 & 20 & 70 & 2 & 180 & 26 & & \\
\hline Schinopsis brasiliensis Engl. & & & & & & & 9 & & & & & \\
\hline Spondias mombin L. & & & & & & & 18 & 2 & & & & \\
\hline Spondias tuberosa Arruda & & & & & & & & & & 4 & & \\
\hline \multicolumn{13}{|l|}{ ANNONACEAE } \\
\hline Annona exsucca Dunal & & & & & 5 & & & & & & & \\
\hline Annona mucosa Jacq. & & & & 1 & & & & & & & & \\
\hline Annona sylvatica A.St.-Hil. & 23 & & & & & & & & & & & \\
\hline \multicolumn{13}{|l|}{ APOCYNACEAE } \\
\hline Aspidosperma cuspa (Kunth) S.F.Blake ex Pittier & & & & 38 & 8 & 3 & & & & & & \\
\hline Aspidosperma cylindrocarpon Müll.Arg. & 2 & 1 & & & & & & & & & & \\
\hline Aspidosperma multiflorum A.DC. & 12 & 1 & & & & & & & & & & \\
\hline Aspidosperma parvifolium A.DC. & 1 & & & & & & & & & & & \\
\hline Aspidosperma pyrifolium Mart. & & & & 120 & 14 & 7 & 12 & 4 & 3 & 15 & 1 & 1 \\
\hline Aspidosperma ramiflorum Müll.Arg. & & & & & & & & 1 & & & & \\
\hline Aspidosperma subincanum Mart. ex A.DC. & & & & & 1 & & 4 & 1 & 1 & & 1 & \\
\hline Aspidosperma tomentosum Mart. & & & & & & & & & & 2 & & \\
\hline \multicolumn{13}{|l|}{ ARALIACEAE } \\
\hline Aralia excelsa (Griseb.) J.Wen & & & & & & & & & & 5 & & \\
\hline Aralia warmingiana (Marchal) J.Wen & 2 & 1 & & & & & & & & & & \\
\hline
\end{tabular}




\section{Família}

Espécie

BIGNONIACEAE

Arrabidaea bahiensis (Schauer) Sandwith \& Moldenke

Handroanthus chrysotrichus (Mart. ex A.DC.)

Mattos

Handroanthus impetiginosus (Mart. ex DC.) Mattos 15

Handroanthus ochraceus (Cham.) Mattos

Handroanthus serratifolius (Vahl) S.O.Grose

Handroanthus spongiosus (Rizzini) S.O.Grose

Jacaranda brasiliana (Lam.) Pers.

Tabebuia roseoalba (Ridl.) Sandwith

Zeyheria tuberculosa (Vell.) Bureau

\section{BORAGINACEAE}

Cordia ecalyculata Vell.

Cordia glazioviana (Taub.) Gottschling \& J.J.Mill.

Cordia incognita Gottschling \& J.J.Mill.

Cordia sellowiana Cham.

Cordia trichotoma (Vell.) Arrab. ex Steud.

BURSERACEAE

Commiphora leptophloeus (Mart.) J.B.Gillet

Protium heptaphyllum (Aubl.) Marchand

Protium sp.

\section{CALOPHYLLACEAE}

Kielmeyera coriacea Mart. \& Zucc.

\section{CANNABACEAE}

Celtis iguanaea (Jacq.) Sarg.

CAPPARACEAE

Cynophalla flexuosa (L.) J.Presl

\section{CELASTRACEAE}

Maytenus rigida Mart.

Maytenus robusta Reissek

Peritassa flaviflora A.C.Sm.

COMBRETACEAE

Combretum duarteanum Cambess.

Combretum leprosum Mart.

Terminalia argentea (Cambess.) Mart.

DILLENIACEAE
$\operatorname{Arcos}(\mathrm{MG})$

Paracatu (MG) Peruaçu (MG)

Coribe (BA)

Arb. Arv. Juv. Arb. Arv. Juv. Arb. Arv. Juv. Arb. Arv. Juv.

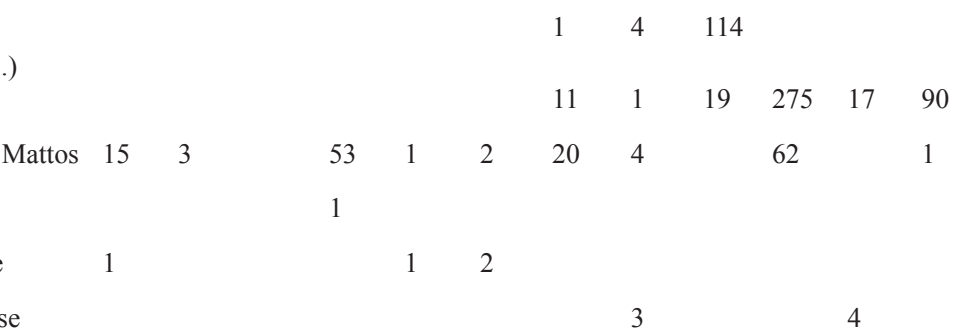

6

1

4

$1 \quad 1$

Curatella americana $\mathrm{L}$.

1 


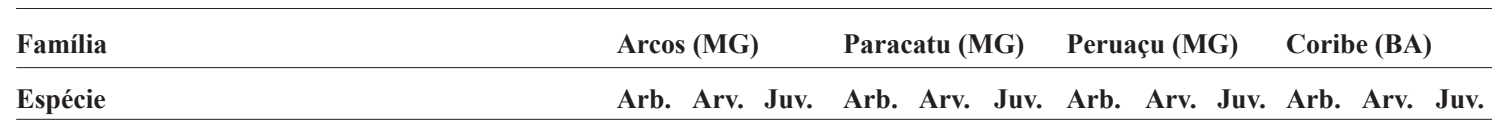

\section{EBENACEAE}

Diospyros coccolobifolia Mart. ex Miq.

9

Diospyros hispida A.DC.

\section{ERYTHROXYLACEAE}

Erythroxylum deciduum A.St.-Hil.

Erythroxylum pelleterianum A.St.-Hil.

Erythroxylum strobilaceum Peyr

EUPHORBIACEAE

Cnidoscolus bahianus (Ule) Pax \& K.Hoffm.

Cnidoscolus oligandrus (Müll.Arg.) Pax

$11 \quad 1$

2

Croton gracilipes Baill.

Jatropha mollissima (Pohl) Baill.

Manihot caerulescens Pohl

Manihot carthaginensis (Jacq.) Müll.Arg.

Sapium glandulosum (L.) Morong

Sebastiania brasiliensis Spreng.

FABACEAE

Abarema langsdorffii (Benth.) Barneby \& J.W.Grimes

4

Acosmium diffusissimum (Mohlenbr.) Yakovlev

Acosmium lentiscifolium Schott

Albizia niopoides (Spruce ex Benth.) Burkart

8

Amburana cearensis (Allemão) A.C.Sm.

Anadenanthera colubrina (Vell.) Brenan

Bauhinia caatingae Harms

Bauhinia cheilantha (Bong.) Steud.

Bauhinia membranacea Benth.

Bauhinia pentandra (Bong.) Vogel

Bauhinia rufa (Bong.) Steud.

Blanchetiodendron blanchetii (Benth.) Barneby \&

J.W.Grimes

99

$\begin{array}{ll}6 & 1\end{array}$

Calliandra foliolosa Benth.

Cenostigma macrophyllum Tul.

Centrolobium tomentosum Guillem. ex Benth.

Chloroleucon dumosum (Benth.) G.P.Lewis

Chloroleucon foliolosum (Benth.) G.P.Lewis

Chloroleucon tenuiflorum (Benth.) Barneby \&

J.W.Grimes

Copaifera langsdorffii Desf.

$10 \quad 42$

$\begin{array}{lll}8 & 13 & 1\end{array}$
1

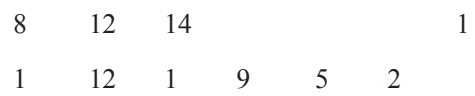

5

$12 \quad 11$

51 $\begin{array}{lll}124 & 39 & 27\end{array}$
112

2

$4 \quad 26$ 


\section{Família}

$\operatorname{Arcos}($ MG)

Paracatu (MG) Peruaçu (MG)

Coribe (BA)

\section{Espécie}

Dalbergia glaucescens (Mart. ex Benth) Benth.

Dalbergia nigra (Vell.) Allemão ex Benth.

Dalbergia villosa (Benth.) Benth.

Dimorphandra mollis Benth.

Dipteryx alata Vogel

Diptychandra aurantiaca Tul.

Enterolobium contortisiliquum (Vell.) Morong

Goniorrhachis marginata Taub.

Hymenaea courbaril L.

$4 \quad 2$

Inga cylindrica (Vell.) Mart.

Lonchocarpus costatus Benth.

Lonchocarpus montanus A.M.G.Azevedo ex M.J.Silva \&

A.M.G.Azevedo

Luetzelburgia auriculata (Allemão) Ducke

Machaerium acutifolium Vogel

Machaerium brasiliense Vogel

Machaerium floridum (Mart. ex Benth.) Ducke

Machaerium hirtum (Vell.) Stellfeld

Machaerium scleroxylon Tul.

Machaerium villosum Vogel

Peltophorum dubium (Spreng.) Taub.

Piptadenia gonoacantha (Mart.) J.F.Macbr.

Platycyamus regnellii Benth.

Platypodium elegans Vogel

Poecilanthe grandiflora Benth.

Poincianella pluviosa (DC.) L.P.Queiroz

Poincianella pyramidalis (Tul.) L.P.Queiroz

Pterodon pubescens (Benth) Benth.

Senegalia polyphylla (DC.) Britton \& Rose

Senegalia tenuifolia (L.) Britton \& Rose

Senna macranthera (Collad.) H.S.Irwin \& Barneby

Swartzia pilulifera Benth.

Sweetia fruticosa Spreng.

ICACINACEAE

Emmotum nitens (Benth.) Miers

7

76

55

Arb. Arv. Juv. Arb. Arv. Juv. Arb. Arv. Juv. Arb. Arv. Juv.

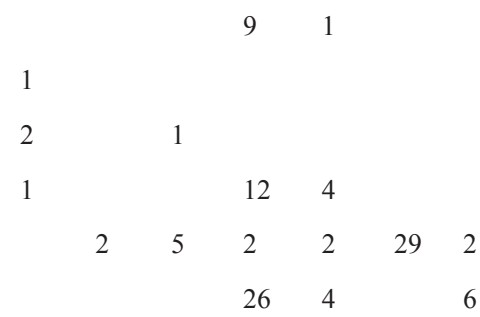

10

$24 \begin{array}{ll}69 & 20 \\ 1 & 1\end{array}$

15

\begin{tabular}{|c|c|c|c|c|c|c|c|c|}
\hline 4 & & 2 & 1 & 8 & & & 5 & 6 \\
\hline & 1 & & & & & & & \\
\hline \multirow[t]{4}{*}{62} & 35 & 18 & 1 & 5 & 22 & 4 & & \\
\hline & & & & 31 & 35 & 87 & & \\
\hline & 14 & & & 2 & & & & \\
\hline & 2 & 3 & & 6 & 2 & 1 & & 2 \\
\hline
\end{tabular}

10

10

4 $3 \quad 3 \quad 3$

\section{LAMIACEAE}

Aegiphila integrifolia (Jacq.) B.D.Jackson

1

146

Vitex polygama Cham.

$30 \quad 1$ 


Família

Arcos (MG) $\quad$ Paracatu (MG) Peruaçu (MG) Coribe (BA)

Espécie

Arb. Arv. Juv. Arb. Arv. Juv. Arb. Arv. Juv. Arb. Arv. Juv.

LAURACEAE

Nectandra lanceolata Nees

1

LOGANIACEAE

Strychnos brasiliensis (Spreng.) Mart.

LYTHRACEAE

Lafoensia vandelliana Cham. \& Schltdl.

MALPIGHIACEAE

Ptilochaeta bahiensis Turcz.

MALVACEAE

Cavanillesia umbellata Ruiz \& Pav.

Ceiba pubiflora (A.St.-Hil.) K.Schum.

Ceiba speciosa (A.St.-Hil.) Ravenna

Guazuma ulmifolia Lam.

2

Helicteres brevispira A.St.-Hil.

Luehea candicans Mart. \& Zucc.

2

Luehea grandiflora Mart. \& Zucc.

Luehea paniculata Mart. \& Zucc.

Pseudobombax marginatum (A.St.-Hil.) A.Robyns

Pseudobombax tomentosum (Mart. \& Zucc.)

A.Robyns

Sterculia apetala (Jacq.) H.Karst.

Sterculia striata A.St.-Hill. \& Naudin

MELIACEAE

Cedrela fissilis Vell.

Trichilia casaretti C.DC.

Trichilia claussenii C.DC.

Trichilia elegans A.Juss.

Trichilia hirta L.

Trichilia pallens C.DC.

MORACEAE

Brosimum gaudichaudii Trécul

Ficus gomelleira Kunth \& C.D.Bouché

Ficus rupicola C.C.Berg \& Carauta

Maclura tinctoria (L.) D.Don ex Steud.

22

2

$21 \quad 8 \quad 6$

1

18

7

4

161

13

11

8

121

$22 \quad 3$

4

MYRSINACEAE

Myrsine umbellata Mart.

3

MYRTACEAE

Campomanesia sessiliflora (O.Berg) Mattos

$\begin{array}{llll}1 & 98 & 25 & 3\end{array}$

Rodriguésia 64(1): A1-A8. 2013 


\begin{tabular}{|c|c|c|c|c|c|c|c|c|c|c|c|c|}
\hline \multirow{2}{*}{$\frac{\text { Família }}{\text { Espécie }}$} & \multicolumn{3}{|c|}{$\operatorname{Arcos}(\mathrm{MG})$} & \multicolumn{3}{|c|}{ Paracatu (MG) } & \multicolumn{3}{|c|}{ Peruaçu (MG) } & \multicolumn{3}{|c|}{ Coribe (BA) } \\
\hline & Arb. & Arv. & Juv. & Arb. & Arv. & Juv. & Arb. & Arv. & Juv. & Arb. & Arv. & Juv. \\
\hline Campomanesia velutina (Cambess.) O.Berg & & 1 & & 15 & 9 & & & & & & & \\
\hline Eugenia dysenterica DC. & & & & 1 & & & & & & & & \\
\hline Eugenia florida DC. & 9 & 11 & 7 & 3 & 40 & 4 & & & & & & \\
\hline Eugenia sp. & & & & & & & & & 2 & & & \\
\hline Eugenia stictopetala DC. & & & & & & & 1 & 1 & & & & \\
\hline Eugenia uniflora L. & 1 & & & & & 1 & & & & & & \\
\hline Marlierea excoriata Mart. & & 2 & & & & & & & & & & \\
\hline Myrcia sp. & & & & 4 & & & & & & & & \\
\hline Myrcia splendens (Sw.) DC. & & 3 & 2 & 1 & & & & & & & & \\
\hline Myrcia tomentosa (Aubl.) DC. & & 3 & 1 & & 4 & & & & & & & \\
\hline Myrciaria cuspidata O.Berg & & & & & & & 11 & 9 & 12 & & & \\
\hline Myrciaria floribunda (H.West ex Willd.) O.Berg & & & & 1 & & & 43 & 6 & 9 & & & \\
\hline Psidium sartorianum (O.Berg) Nied. & & & 2 & & & & & & & & 1 & 1 \\
\hline Psidium sp. & & 3 & & & & & & & & & & \\
\hline NYCTAGINACEAE & & & & & & & & & & & & \\
\hline Guapira opposita (Vell.) Reitz & & & & & & & 1 & & & & & \\
\hline OLACACEAE & & & & & & & & & & & & \\
\hline Ximenia americana $\mathrm{L}$. & & & & & & & & & & 5 & & \\
\hline OPILIACEAE & & & & & & & & & & & & \\
\hline Agonandra brasiliensis Miers ex Benth. \& Hook. & & & & & & & & 1 & & & & \\
\hline PHYLLANTHACEAE & & & & & & & & & & & & \\
\hline Phyllanthus acuminatus Vahl & & & & & 4 & & & & & & & \\
\hline PICRODENDRACEAE & & & & & & & & & & & & \\
\hline Piranhea securinega Radcl.-Sm. \& Ratter & & & & & & & & & & 65 & 5 & 4 \\
\hline POLYGONACEAE & & & & & & & & & & & & \\
\hline Coccoloba obtusifolia Jacq. & & & & & & & 4 & & & & & \\
\hline Coccoloba schwackeana Lindau & & & & & & & 3 & 4 & 2 & & & \\
\hline Triplaris gardneriana Weddell & 1 & & & & & & 13 & & & 40 & & \\
\hline RHAMNACEAE & & & & & & & & & & & & \\
\hline Ziziphus joazeiro Mart. & & & & & & & & & & 1 & & \\
\hline RUBIACEAE & & & & & & & & & & & & \\
\hline Alibertia edulis (Rich.) A.Rich. ex DC. & & & & & & 1 & & & & & & \\
\hline Alseis floribunda Schott & & & & & & & 1 & & & & & \\
\hline Chomelia brasiliana A.Rich. & & 13 & 13 & & & & & & & & & \\
\hline Cordiera sessilis (Vell.) Kuntze & 4 & & 2 & & 2 & 1 & & & & & & \\
\hline Coutarea hexandra (Jacq.) K.Schum. & & & & 4 & 45 & 9 & & & & & & \\
\hline Guettarda sp. & & & & & & & & 1 & & & & \\
\hline
\end{tabular}


Família

Espécie

Guettarda viburnoides Cham. \& Schltdl.

Palicourea crocea (Sw.) Roem. \& Schult.

Randia armata (Sw.) DC.

Simira sampaioana (Standl.) Steyerm.

RUTACEAE

Pilocarpus trachylophus Holmes

Zanthoxylum caribaeum Lam.

Zanthoxylum riedelianum Engl.

Casearia eichleriana Sleumer

Casearia gossypiosperma Briq.

Casearia cf. mariquitensis Kunth

Casearia obliqua Spreng.

Casearia rupestris Eichler

Casearia sylvestris Sw.

Prockia crucis P.Browne ex L.

SAPINDACEAE

Allophylus sericeus (Cambess.) Radlk.

Allophylus sp.

Cupania vernalis Cambess.

Dilodendron bipinnatum Radlk.

Magonia pubescens A.St.-Hil.

Talisia esculenta (A.St.-Hil.) Radlk.

\section{SAPOTACEAE}

Chrysophyllum flexuosum Mart.

Chrysophyllum marginatum (Hook. \& Arn.) Radlk.

Pouteria gardneri (Mart. \& Miq.) Baehni

Pouteria gardneriana (A.DC.) Radlk.

\section{SIPARUNACEAE}

Siparuna guianensis Aubl.

$\operatorname{Arcos}(\mathrm{MG})$

Paracatu (MG) Peruaçu (MG) Coribe (BA)

Arb. Arv. Juv. Arb. Arv. Juv. Arb. Arv. Juv. Arb. Arv. Juv.

1

\section{SOLANACEAE}

Solanum granulosoleprosum Dunal

\section{URTICACEAE}

Cecropia pachystachya Trécul

Urera baccifera (L.) Gaudich. ex Wedd.

VERBENACEAE

Aloysia virgata (Ruiz \& Pav.) A.Juss.

21
20

5

$44 \quad 11 \quad 20$

$2 \quad 11$

Lippia sp. 
Família

$\operatorname{Arcos}($ MG)

Paracatu (MG) Peruaçu (MG)

Coribe (BA)

Espécie Arb. Arv. Juv. Arb. Arv. Juv. Arb. Arv. Juv. Arb. Arv. Juv.

VOCHYSIACEAE

Qualea grandiflora Mart.

Qualea multiflora Mart. 1

Total $635 \quad 316 \quad 234$ 